\title{
Politique
}

\section{Le pouvoir politique et les médias en Amérique Latine}

\section{Oswaldo Capriles}

Volume 1, numéro 2, automne 1982

Les médias et les pouvoirs

URI : https://id.erudit.org/iderudit/040402ar

DOI : https://doi.org/10.7202/040402ar

Aller au sommaire du numéro

Éditeur(s)

Société québécoise de science politique

ISSN

0711-608X (numérique)

Découvrir la revue

Citer cet article

Capriles, O. (1982). Le pouvoir politique et les médias en Amérique Latine.

Politique, 1(2), 69-95. https://doi.org/10.7202/040402ar d'utilisation que vous pouvez consulter en ligne.

https://apropos.erudit.org/fr/usagers/politique-dutilisation/ 


\title{
Le pouvoir politique et les médias en Amérique Latine
}

\author{
Oswaldo Capriles \\ Université Centrale du Vénézuela
}

La constitution historique des systèmes de diffusion de masse en tant qu'appareils $d^{\prime}$ '«industrie culturelle» en Amérique latine:

Tout le monde sait que la formation d'un système d'industrie culturelle, fonctionnant à travers des moyens de diffusion de masse et basée sur les revenus de la publicité, a été l'élément essentiel de la constitution de l'american way of life. On sait aussi que l'accroissement de l'activité publicitaire en termes «exponentiels» est justement dû aux caractéristiques de l'économie de consommation américaine tout comme à la fonction économique et à la structure de leurs grands moyens de diffusion: commercialisation précoce de la presse, constitution d'un schéma de traitement de la radiodiffusion sur la base d'un service commercial privé, puis incorporation de la télévision à ce schéma ${ }^{1}$. On peut également signaler la coïncidence de ces éléments culturels avec le développement industriel, sans précédent sur le plan international, de l'électricité et de l'électronique, l'évolution d'un type de capitalisme en monopole ou oligopole (qui remplacerait la concurrence à l'intérieur du mar-

1. Ce processus est spécialement décrit par Mattelart. 
ché traditionnel par une manipulation du public), l'existence d'une législation particulièrement « libérale » favorisant l'identification de la propriété des médias avec la liberté d'expression, la prédominance à partir de 1940 des agences de presse nordaméricaines, l'hégémonie économico-financière et industrielle des États-Unis à partir de 1920 ainsi que la prédominance politique et le prestige idéologique dérivant de cette hégémonie et du triomphe militaire de la seconde guerre mondiale.

Tous ces facteurs déterminent, entre autres, le contexte dans lequel la publicité nord-américaine a atteint son apogée.

Ce que l'on sait peut-être moins, c'est que ce modèle s'est étendu graduellement au reste du monde après 1930 et beaucoup plus rapidement à partir des années 50 .

Et peut-être n'a-t-on même pas suffisamment insisté sur l'influence prématurée qu'a exercé ce schéma sur l'ensemble des pays au sud du fleuve Rio Grande: ils l'ont suivi aveuglément depuis l'implantation de leurs systèmes de communication institutionnels respectifs (quoique le phénomène de commercialisation de la publicité dans la presse écrite ait été relativement plus tardif qu'aux États-Unis).

De toute façon, l'accent doit être mis sur l'articulation entre la publicité et l'apogée nord-américaine. Cette relation n'est pas dûe au hasard, elle constitue un élément fondamental du système économique, politique et social qui s'instaure, et la nouvelle forme de reproduction de ce capitalisme à tendance monopoliste que l'on voyait déjà se propager à d'autres pays. Il faut également souligner que si l'interconnexion et l'interaction entre les médias commençaient à peine sur le plan de l'organicité, le système des médias aux États-Unis se montrait déjà monopoliste, que ce soit au cinéma dès les années 20, à la radio, ou à la télévision avec la formation des grands networks. De plus, la concentration aura tendance à déborder chaque médium et à connecter certaines industries, telle l'industrie électrique, 
avec l'appareil de diffusion de masse. Ce processus de concentration se montrait fort prudent étant donné les dispositions de la loi anti-trust, mais malgré les limites clairement établies par la Federal Communication Commission (FCC), la radio et la télévision ont bientôt rendu évidente cette tendance à l'interrelation dans le domaine de la diffusion.

Le rapport de la publicité avec les médias, la grande industrie, les entreprises commerciales et les services, est basé sur le rôle même que joue la publicité. Elle intervient de plus en plus entre le producteur et le consommateur, entre la production des biens et la constitution symbolique de la marchandise. La prolifération d'articles sur le marché repose sur une double base: la dilatation de l'acte d'achat et sa constante substitution par des objectifs à court terme, de même que le mythe de la consommation différentielle fondée sur l'acte de la «conspicuous consumption" (telle que définie par Veblen pour une "leisure class) désormais étendue à la grande majorité de la population.

Tel est le schéma qui tend à se constituer à travers le monde à partir du moment où, d'abord le cinéma - au début du siècle - puis la radio et finalement, la télévision, accompagnent mais aussi modifient la presse, constituant ainsi l'induistrie culturelle à laquelle se réfere Théodor Adorno ${ }^{2}$. Cette industrie fabriquera, en énormes doses, une culture de masse pour les foules toujours plus nombreuses des centres industriels et des grandes villes.

2. Il est important de différencier le sens global et analytique du concept d'industrie culturelle utilisé par Adorno et repris par beaucoup d'autres auteurs (Morin, Pasquali, Moles, etc) de celui d'industries culturelles (au pluriel) qui est appliqué par des chercheurs de Grenoble, de la DGRST et de l'Institut National de l'audiovisuel, en France. Le premier prétend rendre compte de la production de messages à travers les médias, en tant que production de culture quotidienne, donc, d'idéologie. Le second se réfere plutôt aux différents processus productifs qui aboutissent à la mise en marché de produits culturels, soient-ils des équipements (hardware), des programmes, des messages ou des choses. 
En Amérique latine, l'appareil de diffusion de masse s'est constitué selon le modèle nord-américain, avec ses conséquences économiques et sociales, cela va sans dire. La phase définitive a débuté dans les années 30 et 40 dans la plupart des pays «avancés» de la région. De puissants appareils d'inculcation se développeront, s'intégreront et s'articuleront avec le système économique de production-commerce-services sous le contrôle principal des transnationales.

Mais c'est avec l'avènement de la télévision et le renforcement du système publicitaire qu'une structure économicopolitique et idéologique s'implantera dès les années 50, dans les pays d'Amérique latine. Cette structure acquiert une rationalité interne de plus en plus grande et implique les propriétaires de médias, les commanditaires, les agences de publicité et les représentants des grandes transnationales. Les commanditaires (sponsors) sont les grands industriels ou commerçants alliés au capital étranger, et dépendant tant pour les brevets que pour le «know-how» des grandes entreprises nord-américaines. Il s'agit surtout de produits de l'industrie légère, de consommation de masse, de services et en particulier, de produits d'usage quotidien: savons, articles de maison, aliments et boissons, etc... Ces produits étant souvent inutiles, c'est précisément la publicité qui en stimule le besoin. Le fait que ce soit les commanditaires qui «investissent» ou dépensent en publicité (plus de 5 milliards de dollars américains en 1980) leur octroie partout en Amérique latine un rôle d'arbitres et maîtres dans la nouvelle société de consommation. Les publicitaires, à l'image des commanditaires dans les pays périphériques, tendent à s'allier ou à être absorbés par les transnationales de la publicité, toutes américaines $^{3}$.

3. Voir notre travail récent pour l'Unesco (à paraître dans le livre préparé à l'occasion de la prochaine Conférence Intergouvernementale Mondiale sur les Politiques culturelles): Systèmes de communication et d'information et valeurs culturelles; le 
De plus, les médias se sont commercialisés; ils dépendent maintenant de la publicité et ont abandonné leurs ambitions politiques de naguère; c'est ainsi que la dépendance économique des pays périphériques se traduit par une dépendance publicitaire, culturelle, informative, mythique, chaque fois plus unifiée, chaque fois plus absorbante. Les mythes, la musique nordaméricaine, les modes de rapports sociaux, les tics, le langage et la mode finissent par s'imposer d'une façon globale et accélérée.

En 1963, Pasquali réalise ce qui semble être le premier essai de caractérisation de la structure du pouvoir des médias, dans son articulation avec les annonceurs et les publicitaires. De plus, son analyse comprenait l'étude des programmations des médias à partir d'une théorie critique originale 4 .

Dans une étude réalisée en 1973 et portant sur cinq pays de la région, Peter Schenkel signalait, dans ses conclusions sur la structure du pouvoir des moyens de diffusion de masse, les similitudes entre les structures économiques de ces pays (Colombie, Chili, Argentine, Pérou et Mexique) ainsi que le rapport de ces similitudes avec le type de schéma implanté dans leur systeme de mass média. Il faisait remarquer le sentiment d'appartenance de classe et les liens des propriétaires des grands moyens de diffusion avec la plupart des entrepreneurs importants, propriétaires ruraux et financiers nationaux et étrangers implantés dans leurs pays respectifs.

L'auteur faisait également ressortir l'utilisation par les propriétaires des médias, de l'influence politique découlant du pouvoir idéologique de ces moyens de communication. L'étude démon-

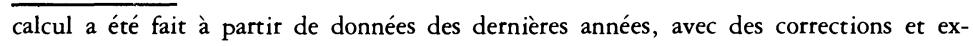
trapolations sur des chiffres officiels.

4. Pasquali, Antonio: Comunicacion y cultura de masas, EBUC, Caracas, 1963 (la première édition de ce livre, peut-être une des plus importantes contributions mondiales à la recherche critique de la communication, est de 1963; depuis, on compte cinq éditions en espagnol et une en portugais au Brésil). 
trait aussi que la concentration de la propriété était une tendance irréversible ${ }^{5}$. Il est intéressant de noter que ces conclusions ressortent en dépit des différences qui caractérisent les régimes politiques de ces pays.

Le rôle des médias dans le développement de la dépendance est fonction de leur propre dépendance. Celle-ci se retrouve à quatre niveaux: la dépendance des nouvelles et de l'information en raison de la prédominance des agences de nouvelles nordaméricaines et occidentales; la dépendance publicitaire en raison toujours de la prédominance des annonceurs ou commanditaires (sponsors) et des agences de publicité transnationales; la dépendance financière, en particulier dans certains médias, à travers la participation de capitaux étrangers (CBS, ABC, NBC, Time-Life, etc...); la dépendance en matière de contenu ou de programmation que l'on retrouve, par exemple, dans la sujétion aux programmes «en boîtes» (séries, feuilletons, spectacles, etc...) produits à l'étranger tant pour la radio que pour la télévision mais aussi au niveau de la production nationale de disques ou encore, d'articles rédactionnels de la presse écrite ${ }^{6}$.

Schenkel cite d'autres facteurs de dépendance,comme le papier journal, les biens d'équipement, le «know-how» relativement sophistiqué employé par la plupart des médias modernes ou les démarches effectuées par des organismes de lobbying, des groupes de pression ou par la Société interaméricaine de presse (S.I.P.) en vue d'assurer la persistance de cette dépendance.

5. Schenkel, Peter: La estructura de poder de los medios de communicaciòn en cinco païses latinoamericanos, ILDIS (Estudios y documentos) Santiago de Chili, 1973.

6. Ibid. 
Les agents intervenant dans la formation du système :

Examinons à titre d'exemple le processus de constitution du système mexicain, décrit par Arriaga (1980), et voyons quels en sont les principaux éléments d'intervention ${ }^{7}$ :

a. l'industrie électronique étrangère intéressée à élargir le marché des appareils en rapport avec la radio, puis avec la télévision.

b. les entreprises du secteur des biens de consommation, en particulier les entreprises étrangères, disposées à financer un système de diffusion de masse qui pourrait commercialiser ses produits, à l'égal du système nord-américain.

c. les entrepreneurs nationaux intéressés à convertir la diffusion de masse en affaire lucrative.

d. les entreprises reliées à la diffusion de masse, soit les agences de publicité, les maisons productrices de films, etc..., également intéressées à élargir le marché pour leurs produits.

La conjugaison de ces facteurs a permis au système mexicain de diffusion de masse de s'intégrer à la dynamique économique par son rapport avec le secteur des biens de consomma$\operatorname{tion}^{8}$.

Le système mexicain de communication de masse a été structuré selon le modèle en vigueur aux États-Unis, en raison de la prépondérance du capital nord-américain dans l'économie nationale. C'est pourquoi les éléments de ce système ainsi que les rapports d'échange sont similaires à ceux observés dans le système nord-américain correspondant ${ }^{9}$.

7. Arriaga, Patricia: Publicidad, economia y comunicacion masiva, CEESTEMNueva Imagen, Mexique, 1980, 276 et ss).

8. Ibid.

9. Ibid. 
L'auteur mentionné ci-dessus tient à relever, dans le schéma de structuration du système mexicain de diffusion de masse, les éléments suivants ${ }^{10}$ :

a. le secteur des biens de consommation, avec la participation du capital étranger, constitue le marché des services offerts par les moyens de communication de masse.

b. les moyens de communication de masse, la plupart entre les mains d'entrepreneurs nationaux, constituent le marché des agences de nouvelles, des maisons de production de films, etc...

c. les agences de nouvelles, nationales et étrangères, vendent leurs services d'information aux mass média.

d. les maisons productrices de programmes, nationaux et étrangers, vendent leur production aux médias de communication de masse.

e. les consommateurs qui, dans un pays périphérique comme le Mexique, ne sont pas la grande majorité de la population, constituent le marché des biens et des services annoncés.

f. la programmation produite ou importée est utilisée par les médias afin d'attirer l'attention du public consommateur ainsi que l'investissement publicitaire des annonceurs, comme aux États-Unis.

Mais passons de la description des éléments à celle de la structure que nous avions ébauchée plus haut.

En Amérique latine, les médias sont liés à plusieurs structures de dépendance ${ }^{11}$. Ils sont d'abord intégrés à la structure

10. Ibid.

11. Les idées ci-devant exposées constituent un résumé des diverses recherches de l'auteur depuis 1969, exprimées notamment dans Capriles, Oswaldo, El Estado y los medios de comunicaciòn en Venezuela, suma éd., Caracas, 1976, La difusion masiva en Venezuela (Partie III d'ouvrage collectif, EUS-UCV, Caracas, 1978). Voir aussi Rapport RATELVE (rapport sur la radio et la télévision pour la Commission Organisatri- 
économique dans la mesure où ils dépendent de la publicité des grands annonceurs ceux-ci étant règle générale rattachés aux entreprises transnationales de l'alimentation, des boissons, des services, des cosmétiques et des biens de consommation durables. À cette dépendance générale s'ajoutent des articulations d'ordre politique et idéologique (Association interaméricaine de radiodiffusion, Société interaméricaine de presse, International Advertising Association etc...).

De plus, la dépendance économique des médias commerciaux entraîne la transmission des stéréotypes dominants d'une culture de consommation dans les systèmes idéologique et culturel des sociétés périphériques. Ce processus de domination est complété par la diffusion d'une échelle de valeurs qui favorise la reproduction de la dépendance économique.

Dans tous les pays de l'Amérique latine la part du secteur privé est plus importante que celle du secteur public en ce qui concerne la propriété et le contrôle des mass média. Cette prédominance est favorisée par les États qui même dans le cas des gouvernements totalitaires, préferent des arrangements mutuellement profitables avec les médias plutôt que d'occuper ce secteur d'activités.

Si l'on observe la structure économique, on peut constater que les médias sont le dernier maillon d'une longue chaîne qui comprend aussi les entreprises publicitaires et les annonceurs. Ce sont les commanditaires qui constituent le moteur du système car ils fournissent l'investissement publicitaire nécessaire à la survie des médias. Les grands commanditaires - ceux qui peuvent se permettre une activité publicitaire constante par

ce du Conseil National de la Culture, Caracas, Suma, 1976), lequel est le produit d'un groupe de travail fonctionnant entre 1974 et 1975, rédigé par trois rédacteurs principaux (Pasquali, Agudo, Capriles) et d'autres rédacteurs associés; de même le récent rapport rédigé par l'auteur pour la prochaine Conférence Intergouvernementale Mondiale des Politiques Culturelles, cit supra. 
la radio, la presse, le cinéma et la télévision - occupent une position dominante dans ce système puisqu'ils déterminent par leurs décisions quels seront les «inputs» de l'appareil publicitaire et des mass média. Ils peuvent aussi définir les règles du jeu et influencer les médias sur les plans politique et idéologique. Les médias sont donc à la fois déterminés par les commanditaires et par les entreprises publicitaires. Ils sont ainsi reliés par des rapports financiers et structurels avec les grandes entreprises financières, industrielles et commerciales ${ }^{12}$.

Les mass média, les agences de publicité et les grands commanditaires forment donc un sous-système dominant à l'intérieur du système des communications de masse en Amérique latine. Mais ces trois composantes du secteur privé se retrouvent, chacun à sa manière dans une situation de dépendance internationale.

Partout en Amérique latine, les grands «commanditaires» ou «acheteurs de publicité» sont de grands industriels, des entreprises de commerce d'importation, des banques et d'autres établissements de crédit. Presque toutes ces entreprises dépendent du financement, de la technologie (patentes, marques, etc...), de l'équipement d'infrastructure, etc..., fournis par les transnationales, et très souvent, sont même des filiales ou des branches de trusts et de holdings étrangers. Il en est de même pour l'activité publicitaire, dans la plupart des pays, les grandes maisons de publicité sont des filiales ou ont des rapports de dé-

12. Ibid. Pour comprendre l'importance du système de l'industrie culturelle/ mass média en Amérique latine, il faut songer à la construction d'une gigantesque économie de consommation de masse, appuyée sur la macrocéphalie urbaine (et donc les migrations, les ceintures de misère, etc...) et le surdéveloppement, aussi artificiel, d'un secteur tertiaire. Le type d' «anti-développement" économique (ainsi que ce processus est qualifié par des auteurs comme Malave Mata au Vénézuela) se constitue donc comme une coupure - si l'on peut s'exprimer dans des termes simplistes - entre une économie de production sous-développée et dépendante et une "économie de consommation" aussi dépendante mais «surdéveloppée», dans des termes relatifs, par rapport à celle-là. 
pendance avec des "grands» des États-Unis (Ogilvy-Matter, Walther Thompson, McCan-Erikson, etc...). De plus, lorsque les transnationales d'aliments, de boissons, etc... possèdent des filiales ou des entreprises dépendantes en Amérique latine, celles-ci ont des contrats publicitaires à long terme avec les "grands» ou alors, elles se hâtent de constituer leurs propres «house agencies» destinées à élaborer exclusivement la publicité pour leurs marques et leurs produits.

On a déjà mentionné les rapports de dépendance entretenus par les mass média de l'Amérique latine avec des entreprises étrangères, mais on doit encore y ajouter les liens qu'ils ont avec les entreprises publicitaires et avec les annonceurs (qu'on vient de décrire), pour ainsi comprendre l'accumulation des rapports de dépendance qui agit sur le produit final du système: le message.

Ainsi, les mass média de l'Amérique latine présentent une double dépendance (interne et externe) qui se traduit par toute une série d'assujettissements: contraintes financières (vis-à-vis de la publicité), contraintes d'équipement et de technologie (face aux entreprises transnationales), et contraintes des contenus (services d'informations, dépêches, photos, etc... pour les journaux, la radio et la télévision/films, programmes, séries, etc... pour le cinéma et la télévision/modèles pour les messages publicitaires, etc..., tout cela est fourni par les transnationales).

Il nous faut donc introduire ici deux précisions dans le modèle: d'abord, la «pyramide» du pouvoir à l'intérieur du système est dominée par le secteur privé; le secteur public, existant à ses côtés, y est subordonné, au moins en tant qu' «émetteur » de messages. En outre, il faut souligner que la pyramide du pouvoir de la diffusion de masse, dans chaque pays, renvoie finalement au véritable pouvoir extérieur, à travers les dépendances qui unissent chaque «étage» ou sous-secteur du secteur privé aux transnationales. 
L'État s'incorpore au système de diffusion:

a. en tant qu'ensemble normatif (corpus des lois, règlements et dispositions qui ont leur base dans les constitutions) toujours sous-tendu par le principe libéral de la «liberté d'expression »;

b. en tant qu'émetteur de messages (propriétaire ou contrôleur de médias qui constituent alors un «secteur public» du système de diffusion) dans le cas où il se réserve une action éducative, culturelle ou informative;

c. et, finalement, au niveau de ses rapports de force avec le secteur privé (rapports économiques, politiques et autres, formels ou informels). Pour les besoins de cet exposé, la fonction normative sera laissée de côté alors que la fonction d'émission et les rapports public-privé seront rapidement décrits ${ }^{13}$.

\section{L'État en tant que communicateur (secteur public):}

Parallèlement aux entreprises privées, l'État peut constituer son propre système d'information et de diffusion, établir des réseaux ou simplement maintenir certains services de communication, il peut aussi s'abstenir d'exercer un rôle dans la communication sociale. Lorsqu'il joue un rôle actif celui-ci peut être exercé directement à travers des Ministères ou des bureaux spécialisés ou bien au moyen d'entreprises ou d'institutions ad boc.

Mais quelque soit la stratégie de diffusion choisie, dans la plupart des pays d'Amérique latine, le secteur public occupe une position minoritaire dans le système de diffusion de masse. Même s'il y a suprématie du secteur privé sur le secteur public,

13. Ibid. En général, la situation ici décrite est celle de la plupart des pays de l'Amérique latine, telle que nous l'avons rencontrée partout. 
la relation entre les deux n'est pas stable et constitue un terrain riche en contradictions.

L'État dans ses rapports avec le secteur privé:

Le champ de la communication de masse pose la question de la responsabilité politique qui incombe à l'État quant aux formes, contenus, effets et autres aspects du processus de diffusion, soumis de par les Constitutions et les lois des différents pays à un contrôle plus ou moins poussé selon les besoins et les objectifs de l'action gouvernementale. C'est l'aspect le plus important dans les pays où le secteur privé est hégémonique en ce qui concerne la capacité et le pouvoir de diffusion. En effet, l'État peut se démettre d'un rôle actif (communicateur) mais ne peut éviter d'être inséré dans le champ des responsabilités sociales de la communication, soit d'un point de vue négatif (interdictions ou limitations destinées à la protection de la société ou des individus), soit d'un point de vue positif (contenus éducatifs ou culturels obligatoires, proportions accrues de messages en relation avec les besoins réels de la population, etc...).

Mais en plus des fonctions de fiscalisation et de contrôle, l'État peut avoir des rapports économiques avec le secteur privé: il s'agit des «politiques» de stimulation, de promotion ou d'encouragement de certaines branches de la communication (cinéma national, journaux provinciaux, édition de livres, médias culturels, revues, etc...), ainsi que des prêts, subsides ou subventions accordés à certains médias par des entités publiques, y compris des aides indirectes quelquefois fondées sur des subterfuges politiques (non-récupération du crédit ou des prêts accordés, impôts et taxes trop faibles ou non recouvrés, achat d'entreprises en faillite pour venir en aide aux propriétaires) ${ }^{17}$.

Or, c'est justement au niveau des rapports réels entre le secteur privé et la structure globale de l'État, que nous avons

14. Ibid. 
trouvé dans la plupart des pays et sous des gouvernements d'orientation différente, maintes preuves de la faiblesse communicationnelle foncière et voulue du secteur public, et d'un courant permanent d'aide, de promotion ou de soutien du secteur public au secteur privé des médias. Les gouvernements accordent des prêts, des subsides et d'autres aides financières aux médias qui leur sont les plus proches; généralement, le secteur public est un des plus importants «commanditaires» de publicité ou propagande, qu'il doit répartir entre les médias privés, sous peine d'être accusé de favoritisme ou d'atteinte à la liberté d'expression.

L'État est donc d'une certaine façon «prisonnier» du mythe de la liberté d'expression, à travers l'existence d'un secteur privé surdéveloppé qui domine le terrain de la diffusion de masse partout en Amérique latine, à partir d'un processus historique irrésistible, souvent grâce à la protection et à l'appui financier des gouvernements et, en général, grâce à des dépenses assez importantes faites par ces mêmes gouvernements lors des campagnes de propagande conçues par des entreprises publicitaires et véhiculées par les médias privés. Mais la dépendance «politique-idéologique» de l'appareil d'État et des gouvernements est plus importante comparativement à la dépendance relative du secteur privé concernant le financement de source étatique. Il s'agit, donc, de relations asymétriques qui favorisent finalement le secteur privé, en tant que «fer de lance» des groupes économiques dominants dans chaque formation sociale.

On peut dire que l'État dispose d'une faible «autonomie relative " face au secteur privé qui domine le système de diffusion de masse, encore plus relative que celle qu'il possède dans d'autres aires d'action, où l'élite économique accepte et quelquefois même appelle l'intervention de l'État. Celui-ci d'ailleurs, dépense moins dans la création ou le développement de ses propres services d'information 
et de diffusion, que dans des activités publicitaires confiées au secteur privé ${ }^{15}$.

Pour donner la mesure de la situation actuelle, il faudrait encore, rappeler l'intensification du processus de concentration financière internationale qui s'est produite à partir des années 60 , et qui s'est accrue avec le développement de l'électronique, des télécommunications et de l'informatique (y compris la consolidation d'une «télématique », d'une « robotique», d'une «bureautique», et même d'une «télémédiatique», ainsi que d'un «logiciel») de plus en plus sophistiqué. Le développement du «quatrième secteur» des économies «post-industrielles» analysé par E. Parker et d'autres, dès 1969, est une situation lourde de conséquences pour le tiers-monde: la dépendance technologique s'est accrue à une vitesse sidérale, et les autres dépendances se sont par conséquent multipliées dans la même proportion.

Mais nous nous limiterons à rappeler ici les résultats des recherches concernant un des domaines de la dépendance concrète des médias en Amérique latine: la dépendance informative, c'est-à-dire, les sources de l'information sous forme de dépêches, articles, actualités, etc...

Les nouvelles (l'information) en Amérique latine:

En 1966, le journaliste Eleazar Diaz-Rangel publie une étude dénonçant la manipulation et le déséquilibre qui caractérise le flux de nouvelles et d'informations qui finit par se déverser sur les publics latino-américains à travers les médias. Entre autres points importants, cette étude rapporte une analyse effectuée par l'auteur et portant sur les journaux «les plus lus » par les élites économiques et politiques de l'Amérique latine ${ }^{16}$.

15. Ibid.

16. Diaz-Rangel Eleazar, Pueblos sub informados (Peuples sous-informés), 1966, ré-édité en 1976 avec l'addition d'un prologue qui fait un "state of the art» du sujet, 10 ans après cette étude "pionnière » en Amérique latine. 
Une liste de journaux «les plus représentatifs» en Amérique latine fut établie en s'inspirant d'enquêtes antérieures et d'une classification choisie pour Time $\mathcal{E}$ Life par une entreprise de New York à partir d'une enquête réalisée auprès de 216 cadres. Selon l'enquête mentionnée, les 21 quotidiens les plus lus par les élites économiques et les fonctionnaires étaient les suivants:

Journaux les plus lus par les Gérants, Cadres, Fonctionnaires et Agents des plus importantes Compagnies de distribution établies

Nombre de personnes $\%$

Excelsior (Mexique)

Miami Herald

O Estado (Sao Paulo, Brésil)

New York Times

El Universal (Caracas Vla)

La Nacion (Argentine)

La Prensa (Pérou)

El Tiempo (Colombie)

El Comercio (Pérou)

O Globo (Brésil)

The Wall Street Journal

El Mercurio (Chili)

La Nacion (Costa Rica)

Novedades (Mexico)

San Juan Star (Puerto Rico)

El Espectador (Colombie)

La Estrella (Panama)

El Mundo (Puerto Rico)

El Nacional (Vénézuela)

The News (Mexique)

La Prensa (Argentine)

Se basant sur l'enquête ci-haut mentionnée et introduisant des modifications bien justifiées, Diaz Pangel a choisi les 14 journaux qui lui ont semblé les plus importants. Ceux-ci sont les suivants: 
14 Grand Journaux Latino-Américains

\begin{tabular}{|c|c|c|c|c|}
\hline & Fondé en & Fondateur & Directeur & $\begin{array}{c}\text { Nombre de } \\
\text { pages }\end{array}$ \\
\hline El Universal (Caracas) & 1909 & Andres Mata & Luis Todilfo Nunez & 60 \\
\hline El Nacional (Caracas) & 1943 & H. Otero V. & Ramon J. Velazquez & 44 \\
\hline El Tiempo (Bogota) & 1911 & & R. Garcia Pena & 28 \\
\hline El Espectador (Bogota) & 1887 & & Gabriel y Gmo Cano & 22 \\
\hline Excelsior (Mexico) & 1917 & R. Alducin & M. Becerra Acosta & 46 \\
\hline Novedades (Mexico) & 1936 & & Ramon Beteta & 38 \\
\hline Diario de Noticias (Rio) & 1930 & O. Dantas & O.J. Portelas Dantas & 24 \\
\hline O Globo (Rio) & 1925 & I. Marinho & Roberto Marinho & 40 \\
\hline El Comercio (Quito) & 1906 & C. Mantilla & Carlos Mantilla & 28 \\
\hline La Nacion (Buenos Aires) & 1870 & B. Mitre & Dr. B. Mitre & 26 \\
\hline La Prensa (Buenos Aires) & 1869 & Jose C. Paz & Alberto Gainza P. & 24 \\
\hline El Comercio (Lima) & 1839 & & Luis Miro Quesada & 28 \\
\hline El Mercurio (Santiago) & 1827 & A. Edwards & & 54 \\
\hline La Maǹana (Montevideo) & 1917 & P. Manini Rios & Carlos Manini Rios & 24 \\
\hline
\end{tabular}

Le contenu de ces journaux a été examiné le 30 juin 1965. Les résultats ont été significatifs quant à la dépendance par rapport aux sources de nouvelles nord-américaines et transnationales. La quantité d'informations sur les États-Unis était tout à fait disproportionnée, même en comparaison avec l'espace réservé par les autres journaux dans le monde à toute l'information émanant de l'étranger ${ }^{17}$.

Les nouvelles concernant le Tiers-Monde non latino-américain étaient en minorité. Des 532 dépêches publiées ce jourlà, 13 seulement avaient trait à ce secteur. Par contre, 55 pour cent des nouvelles se référaient aux pays industrialisés (Europe et États-Unis). Quant à l'aspect thématique, le domaine "États-Unis» équivalait sensiblement celui de l'ensemble de l'Amérique latine. Certains journaux très importants, comme l'Excelsior du Mexique, consacrait 18 pour cent de son information aux États-Unis, contre 8 pour cent à l'Amérique latine. Ceci était aussi valable pour un autre «grand quotidien» mexicain, Novedades. 
Les deux faits les plus saillants présentés dans ces journaux comme des grandes nouvelles d'ordre général, étaient une offensive conjointe des États-Unis et de leurs alliés au Vietnam et la visite d'un couple royal au Parlement hollandais. Les quotidiens mentionnés publiaient peu de nouvelles au sujet de leurs propres réalités. La crise dominicaine et l'intervention américaine, le coup d'État militaire écuatorien ou encore un combat de «guerilleros» au Vénézuela demeuraient des informations marginales reléguées au troisième plan et ne faisant généralement pas la manchette dans les pays d'origine de ces événements ${ }^{18}$.

Les régions d'origine de l'information (c'est-à-dire, les lieux où se sont déroulés les événements rapportés) étaient ainsi distribuées :

\begin{tabular}{rrrrrc} 
U.S.A. & Amérique Latine & Europe & Asie & Afrique & Autres \\
13 & 30 & 15 & 4 & 4 & 2 \\
11 & 18 & 16 & 2 & 1 & - \\
8 & 5 & 7 & 2 & 2 & - \\
8 & 7 & 4 & 2 & 1 & - \\
18 & 8 & 13 & 4 & 3 & 2 \\
14 & 8 & 16 & 2 & 3 & - \\
1 & 4 & 6 & 2 & 2 & - \\
8 & 6 & 13 & 4 & 2 & 1 \\
14 & 21 & 11 & 3 & 2 & 3 \\
4 & 4 & 10 & 2 & 1 & - \\
6 & 11 & 8 & 2 & 1 & - \\
9 & 10 & 9 & 3 & - & - \\
10 & 9 & 26 & 6 & 7 & - \\
9 & 11 & 14 & 2 & 1 & - \\
\hline 132 & 154 & 168 & 40 & 30 & 8 \\
\hline $24 \%$ & $28 \%$ & $31 \%$ & $7 \%$ & $5 \%$ & $1 \%$
\end{tabular}

Le contrôle des agences de nouvelles transnationales sur ces journaux et en particulier, la prédominance nord-américaine ont été mis en relief de la façon suivante ${ }^{19}$ :

18. Ibid.
19. Ibid.

El Universal

El Nacional

El Tiempo

El Espectador

Excelsior

Novedades

Diario de Noticias

Ô Globo

El Comercio (Q)

La Nacion

La Prensa

El Comercio (L)

El Mercurio

La Maǹana 
Origine de l'information par dépêches Agences (cm $\times$ col.)

\begin{tabular}{|c|c|c|c|c|c|c|}
\hline & $U P I$ & $A P$ & Reuter & $A F P$ & Ansa & Autres \\
\hline El Universal & 507 & & & 209 & & 69 \\
\hline El Nacional & & 545 & & 150 & & 14 \\
\hline El Tiempo & 83 & 92 & & 85 & & \\
\hline El Espectador & 322 & & & & & 35 \\
\hline Excelsior & & 455 & & 109 & & 18 \\
\hline Novedades & 102 & 295 & & 138 & & 23 \\
\hline Diario de Noticias & 94 & & & & 15 & 15 \\
\hline Ô Globo & 124 & 125 & & 94 & & \\
\hline El Comercio (Q) & 540 & & & 140 & & 30 \\
\hline La Nacion & & 186 & & 61 & & 40 \\
\hline La Prensa & 544 & & & & & \\
\hline El Comercio (L) & 227 & & 57 & 63 & 3 & \\
\hline El Mercurio & 244 & 220 & 58 & 110 & & 152 \\
\hline \multirow[t]{3}{*}{ La Maǹana } & & 217 & & 114 & 69 & 30 \\
\hline & 2783 & 2135 & 115 & 1273 & 87 & 426 \\
\hline & $40,8 \%$ & $31,3 \%$ & $1,6 \%$ & $18,6 \%$ & $1,2 \%$ & $6,2 \%$ \\
\hline
\end{tabular}

Les agences nord-américaines UPI et AP avaient fourni 72 pour cent de l'information internationale ce jour-là dans les quotidiens sélectionnés. La principale source était l'AP (Associated Press avec 40,8 pour cent du total publié alors que la UPI représentait 31,3 pour cent.

Par ailleurs, la publicité occupait un espace bien supérieur à l'espace rédactionnel de l'information internationale, et encore plus grand par rapport aux nouvelles vraiment importantes pour les intérêts et l'avenir de la région latino-américaine. Une comparaison du tableau suivant avec le tableau antérieur permettra de constater entre autres, une des graves conséquences de la dépendance publicitaire. 
Volume d'un jour de publicité 20 (en centimètre $\times$ colonne)

$\begin{array}{cccc} & \text { Entreprises et } & \text { Entreprises et } & \text { Entreprises et } \\ \text { produits } & & \text { produits } & \text { produits } \\ \text { Total nationaux } & \text { Officiel Spectacle } & \text { U.S.A. d'autres pays }\end{array}$

\begin{tabular}{lrrrrrr} 
El Universal & 16.759 & 10.590 & 1.324 & 1.500 & 2.305 & 1.040 \\
El Nacional & 10.217 & 5.706 & 1.115 & 1.320 & 1.525 & 551 \\
El Tiempo & 5.673 & 2.780 & 104 & 800 & 1.489 & 500 \\
El Espectador & 4.608 & 1.978 & 170 & 880 & 1.224 & 356 \\
Excelsior & 12.332 & 8.719 & 230 & 1.500 & 1.058 & 815 \\
Novedades & 7.180 & 4.104 & 160 & 1.300 & 790 & 826 \\
Diario de Noticias & 2.631 & 936 & 150 & 980 & 435 & 130 \\
Ô Globo & 6.224 & 4.585 & 20 & 364 & 745 & 510 \\
El Comercio (Q) & 4.856 & 2.240 & 184 & 884 & 985 & 563 \\
La Nacion & 6.310 & 5.659 & 60 & 340 & 205 & 46 \\
La Prensa & 5.116 & 4.658 & 50 & 50 & 250 & 108 \\
El Comercio (L) & 5.026 & 2.870 & 235 & 410 & 700 & 811 \\
El Mercurio & 13.288 & 9.097 & 972 & 870 & 1.233 & 1.116 \\
La Manana & 3.162 & 2.242 & 200 & 100 & 275 & 345 \\
& & & & & & 7.717 \\
\hline & 103.372 & 66.164 & 4.974 & 11.298 & 13.219 & $7 \%$
\end{tabular}

Dans un relevé critique sur la recherche latino-américaine, les chercheurs Beltran et Fox rappellent les conclusions similaires à celles de Diaz Rangel présentées dans d'autres études comme celle du CIESPAL (Quito) l'année suivante (1967) et celle de Rayes Matta (1976). Reyes Matta a apporté un ensemble de preuves concernant les dépêches non publiées en Amérique Latine, particulièrement celles en rapport avec d'importants événements de la région; il conclut qu'il existe une évidente autocensure ou «manipulation» de l'information, comme il préfere la qualifier, sans arriver à déterminer la cause ${ }^{21}$.

En 1977, Luis Anibal Gomez a essayé de déterminer plus précisément le caractère de cette manipulation, et de situer le

20. Ibid.

21. Ibid. 
rôle des «gatekeepers». Cette investigation, centrée sur le cas du Vénézuela, arrivait à la conclusion que les agences internationales de nouvelles conditionnent l'image que les pays latinoaméricains ont des autres pays, y compris de leurs voisins immédiats. Le "gatekeeper» transnational, selon Gomez, «jouit de plus grandes occasions pour conditionner que le gatekeeper local». Il a aussi constaté que les agences internationales véhiculent peu d'informations sur le Tiers-Monde non latinoaméricain $^{22}$.

La prédominance des agences de nouvelles nord-américaines est un reflet de l'hégémonie économique et politique des États-Unis dans cette région du monde. Cette conclusion se voit renforcée par le traitement de faveur que les journaux accordent à ces agences, les survalorisant comme des sources dignes de confiance. Les nouvelles de l'Amérique latine qui s'exportent sont généralement du genre «spotnews", c'est-à-dire des informations ponctuelles, hors contexte, généralement scandaleuses ou de type «faits divers».

De cette façon, les stéréotypes propres à la culture nordaméricaine et européenne sont appliqués à chaque pays: le Vénézuela est ainsi présenté comme un pays de pétrole et de sports et rien d'autre, sauf lorsqu'il y a des catastrophes et des scandales. Une fois de plus, la principale critique, à notre avis, s'adresse aux médias eux-mêmes car ceux-ci n'utilisent pas une pluralité de sources d'information et se soumettent à la domination des agences nord-américaines ${ }^{23}$.

22. Beltran, L. Ramiro et Fox, Elizabeth: Mass média et domination culturelle incorporé dans le dossier sur Mass média, éducation et culture de la revue Perspectives, Unesco, n. 1, 1980. (vol. X); Reyes Matta, Fernando (éd.): La informaciòn en el nuevo orden internacional, ILET, Mexique, 1977. Un travail de Diaz Rangel dénonçait déjà en 1972 le système de censure-auto-censure des nouvelles dans les journaux (Noticias censuradas, Caracas, 1974).

23. Gomez, L.A.: La circulaciòn de la informaciòn noticiosa, el caso de Venezuela, UNESCO/Ininco, Caracas, 1977. 
Il n'existe pas de traitement de la dépêche par la rédaction en chef des journaux, ce qui aurait pu contribuer à restituer un certain équilibre et une certaine compréhension de l'information. Pire encore: Gomez conclut que le «gatekeeper» local restitue la dépendance du journal par rapport à la nouvelle nord-américaine alors que les agences nord-américaines ellesmêmes ont valorisé une nouvelle latino-américaine. Cela veut dire qu'on «corrige» les agences de nouvelles nord-américaines quand celles-ci ne sont pas suffisamment "pro-américaines» 24 .

La plupart des travaux, comme ceux de Reyes Matta et celui des frères Abreu (celui-ci concerne un jour de l'année 1976, à Caracas), ou encore celui du CIESPAL (sur deux semaines de presse en Amérique latine en 1967), s'accordent à reconnaître le fait de la dépendance dans la nouvelle, de la responsabilité qui incombe aux journaux eux-mêmes, et de la méconnaissance mutuelle entre les pays de la région comme fruit de cette dépendance (Reyes Matta a signalé dans son étude le cas éloquent du Surinam dont l'indépendance est passée presque inaperçue $)^{25}$.

Comme l'a signalé Pasquali, le «collaborateur local» est généralement «plus catholique que le Pape» et défend avec d'autant plus de force les intérêts de la puissance hégémonique et ceux des réseaux d'information transnationaux que sa propre carrière professionnelle est évidemment liée à la fois aux grandes entreprises et aux élites du pays. Et les intérêts de ses patrons sont reliés pour des raisons de simplification commerciale, de maximisation du gain et de conformisme réactionnaire, de minimisation de l'effort et de l'investissement, aux grands réseaux ou entreprises de type transnational fournissant de l'information, des programmes, des modèles publicitaires, etc...

24. Voir Gomez, Reyes Matta, Beltran, etc...

25. Telle est la plus importante conclusion du travail cité de L.A. Gomez. 
Un nombre élevé d'études, non seulement critiques mais aussi prospectives, se sont ajoutées aux enquêtes classiques sur le déséquilibre dans l'information en Amérique latine. Cellesci ont surgi en particulier à la suite de la parution accrue de thèmes concernant le Nouvel Ordre International de l'Information, suscité par les Conférences de Bogota et de Costa Rica sur les politiques nationales de communication. L'organisme ILET de Mexico a publié plusieurs travaux et compilations sur ces sujets, réunissant des travaux de Reyes Matta, de Somavia, de Schiller, d'Ordonez, de Encalada, de L.A. Gomez, de Bonilla de Ramos, etc... ${ }^{26}$

Il faudrait encore rappeler quelques constantes de l'action combinée des agences transationales d'information, spécialement l'UPI et l'AP et des "gatekeepers » de la presse:

- Dans le cas des journaux et des actualités des pays dits "développés" il est très clair que tout conflit nord-sud va être interprété et manipulé comme s'il s'agissait d'un conflit est-ouest. Ceci est parfaitement évident dans toutes les nouvelles concernant les mouvements de libération nationale ou de protestations, les manifestations populaires ou les actes de gouvernements considérés comme «hostiles» aux intérêts économiques des transnationales et donc, contraires au pouvoir politico-militaire des États-Unis. Dans ce sens, il est saisissant de constater la différence du traitement donné, d'une part, aux événements polonais, et d'autre part, aux événements de l'Amérique centrale (plus frappant encore, étant donné les milliers de morts laissés par des «escadrons» d'assassins clairement soutenus par les dictatures militaires ou paramilitaires du Guatemala et du Salvador).

26. Le travail d'Abreu et Abreu (Caracas, 1977) se proposait de reprendre le travait fait par Diaz Rangel 10 ans avant, afin de mesurer les changements: la situation a été trouvée aussi négative que celle décrite par les autres travaux sur la question. Voir aussi La noticia internacional, publié par ILET (1977), avec de nombreuses collaborations. 
- La fragmentation dans le temps et dans l'espace est un des processus courants de la grande presse, que ce soit celle des pays «développés» ou celle du Tiers-Monde, cette fragmentation implique toujours une décontextualisation de l'événement, une amnésie des facteurs et des causes historiques et sociales qui seuls pourraient l'expliquer et le faire devenir matière à réflexion.

- La simplification et la généralisation abusives sont deux procédés, en apparence contradictoires, mais en réalité complémentaires, que l'on utilise pour modifier délibérément la signification des événements.

Pour finir un exposé qui risque de devenir trop long, je voudrais apporter quelques illustrations de l'action de la grande presse internationale et des agences transnationales de dépêches:

a. Il y a le cas très connu de l' «intoxication » informative menée par les agences américaines, spécialement l'UPI, contre le gouvernement de l'unité populaire au Chili. Cette agence alla jusqu'à déclarer que l'avion conduisant Allende en visite officielle en Colombie, était chargé d'armes pour les guerilleros comlombiens! (ce qui a conduit le gouvernement chilien à fermer l'UPI quelques jours).

b. La campagne menée dans la plupart des grands journaux du monde contre la Conférence des Ministres de l'Amérique latine sur les politiques de communication, tenue à San José de Costa Rica en 1976, campagne qui a même lancé de fausses nouvelles (existence d'un «plan» russe, maladie du délégué général de l'Unesco) et qui a duré plusieurs mois, avec des articles et des insertions diverses dans plus de 200 médias dans le monde, pour un total de plus de 700 insertions ${ }^{27}$.

27. Beltran, L. R.: Latin America and the U.S.: flaws in the free flow of information, dans Richstad $\mathrm{J}$ : «New perspectives in international communication" (EastWest Center, Honolulu, 1976). Voir travaux récents de Reyes Matta dans revue UNDAAL et dans Comunicacion y cultura. 
c. La victime la plus récente de ces fausses nouvelles systématiquement produites est le Nicaragua: ainsi la fameuse photo des indiens miskitos publiée par le Figaro et reprise par le Département d'État américain, ainsi que la présentation d'un «déserteur» nicaraguayen, (qui n'était qu'un simple étudiant), des photos et des «renseignements» sur des campements militaires, etc... Ces «informations» ont été recueillies et diffusées par plusieurs médias en Amérique latine; et les rectifications n'ont pas été apportées depuis... 28

28. Voir mon travail Actions and reactions to communication policies within the framework of Unesco: analysis of the Costa Rica Conference (paper presented to Seminar on international communication and third world participation: A conceptual and practical framework, 1977; publié aussi en espagnol dans: El desafio juridico de la informaciòn internacional, ILET, Nueva Imagen, Mexique, 1979). Il est clair que, dans le cas du Nicaragua, les "nouvelles» étaient directement manipulées par le Département d'État américain et la C.I.A., mais il n'est pas moins clair que certains mass média ont «suivi» plus que d'autres: ainsi Newsweek a même illustré sa couverture avec les photos des camps militaires nicaraguayens (la disposition des lieux étant une "preuve» de l'influence soviétique!), ou le Figaro qui est même arrivé, sans le vouloir, à faire tomber les autorités américaines dans le piège en utilisant une photo de l'époque du dictateur Somoza comme si elle avait été prise ensuite. De même que le cas du faux «déserteur» qui a tourné en ridicule les affirmations du Département d'État. Pourtant une bonne partie des publics touchés par ces «informations» n'ont jamais «reçu» de rectification quant aux «faits» en cause...

Dans le cas très récent du conflit des Îles Malouines, il est très intéressant de constater l'absence de contexte historique au problème, ainsi que celle du contexte géographique quant à la très faible "autonomie" économique des îles qui comme les Malouines (et tant d'autres anciennes possessions coloniales conquises par la force) ne constituent que des enclaves en partie abandonnées par l'ancienne force coloniale, sous-peuplées et dépendantes des pays riverains plus roches. L'on voit ainsi, que le problème des «titres» historiques n'a pas été évoqué, sauf par une revue française (le Nouvel Observateur) et encore sur un ton mi-ironique, visant plutôt l'anecdote, à propos de la souveraineté franco-espagnole originelle. Newsweek, pour sa part, a publié un encadré (mais séparé du gros des informations et aussi sur un ton anectotique) dans lequel on apprend que la Société anglaise propriétaire de presque la moitié de la surface utile des îles et de la presque totalité du cheptel, a été sur le point de vendre ses actifs à un millionnaire argentin, mais le gouvernement anglais s'y est opposé. Pourtant le même gouvernement a patronné un rapport qui recommande de rapprocher les îles de l'Argentine, vue la distance et les difficultés économiques d'un "pouvoir à distance " comme celui de l'Angleterre. 
d. Il faudrait aussi rappeler de quelle manière toute la presse occidentale a attribué aux pays de l'OPEP, la responsabilité exclusive des difficultés économiques des pays occidentaux et même, de la recrudescence de la misère au TiersMonde, en se gardant en même temps de signaler le rôle de la politique monétaire américaine, de la spéculation boursière et financière internationale, de la concurrence entre les pays industriels, et encore moins de pointer les véritables responsables quant à la misère de la plupart des régions du Tiers-Monde, ou encore de mentionner l'échange inégal et les bas prix des matières premières, ou les énormes bénéfices des transnationales sur la main-d'œuvre et les produits du Tiers-Monde...

Les procédés et les exemples que nous venons d'exposer ne font pas seulement partie du problème de la communication internationale et de celui de l'image que se font les pays industrialisés des régions "sous-développés", mais aussi du probleme interne de l'information dans les pays $d u$ Tiers-Monde eux-mêmes. C'est justement le but de cette «action combinée » à laquelle nous avons fait allusion dans les lignes précédentes, que de produire une même image, déformée et manipulée, et de l'adresser à tous les publics du monde. Donc, il s'agit bien d'une image universelle des rapports économiques, politiques et sociaux, nationaux et internationaux, qui est imposée à la fois dans les sociétés «développées» et «sous-développées».

Finalement, ces «informations» fabriquées, ces «interprétations » mensongères ou abusives, ces "événements " déformés, restent comme des vérités, deviennent des «acquis » d'une "réalité » construite de toutes pièces: les clarifications, les rectifications, les déclarations de savants, d'hommes de bonne volonté, même les scandales lorsqu'on découvre des activités frauduleuses de la part d'agences de presse ou des médias, n'arrivent jamais 
à effacer le mal qui a été fait: c'est là justement le chef d'accusation le plus grave contre le système actuel de l'information internationale. 